\title{
Impactos da miopia no desenvolvimento cognitivo da criança: uma revisão narrativa
}

\author{
Impacts of myopia on child cognitive development: a narrative review
}

Impactos de la miopía en el desarrollo cognitivo infantil: una revisión narrativa

\begin{abstract}
Emilio Rintaro Suzuki Junior ${ }^{2}$, Alice Pinheiro Vanetti ${ }^{1}$, Bianca Carollyne Martins Pinto ${ }^{1}$, Geovana do Nascimento Almeida ${ }^{1}$, Laryssa de Lacerda Rodrigues ${ }^{1 *}$, Leonardo Mendonça Monteiro de Castro ${ }^{1}$, Natalia Batista Zanetti' ${ }^{1}$.
\end{abstract}

\section{RESUMO}

Objetivo: Identificar, por meio de uma revisão de literatura, a existência de importantes prejuízos ao desenvolvimento intelectual da criança portadora de miopia, reafirmando a necessidade do diagnóstico e do tratamento precoces. Revisão bibliográfica: A miopia é caracterizada pela incapacidade do olho de acomodar a luz direcionada a ele na própria retina, formando a imagem anteriormente, e dificultando a visão distante dos objetos. Essa patologia, possui significativa prevalência na infância, constituindo um dos importantes fatores para baixa acuidade visual. Paralelamente, os primeiros anos de vida são os mais importantes para o desenvolvimento da criança, dessa forma a baixa acuidade visual pode comprometer o desenvolvimento neuromotor, das habilidades manuais, da linguagem e da cognição. Considerações finais: O desenvolvimento das competências intelectuais da criança é um processo dependente das informações sensoriais recebidas através da visão. Desse modo, identificar a baixa acuidade visual em escolares, especificamente a miopia, torna- se essencial para seu desenvolvimento cognitivo adequado.

Palavras-chave: Criança, Miopia, Cognição.

\begin{abstract}
Objective: To identify, thought a literature review, the existence of significant damage to the intellectual development of children with myopia, reaffirming the need for early diagnosis and treatment. Bibliographic review: Myopia is characterized by the inability of the eye to accommodate the light directed to it on the retina itself, forming the image anteriorly, and making distant vision of objects difficult. This pathology has a significant prevalence in childhood, constituting one of the important factors for low visual acuity. At the same time, the first years of life are the most important for the child's development, thus, low visual acuity can compromise neuromotor development, manual skills, language and cognition. Final considerations: The development of a child's intellectual skills is a process dependent on sensory information received through vision. Thus, identifying low visual acuity in schoolchildren, specifically myopia, becomes essential for their proper cognitive development.
\end{abstract}

Keywords: Child, Myopia, Cognition.

\section{RESUMEN}

Objetivo: Identificar, a través de una revisión de la literatura, la existencia de daño significativo al desarrollo intelectual de los niños con miopía, reafirmando la necesidad de un diagnóstico y tratamiento precoces. Revisión bibliográfica: La miopía se caracteriza por la incapacidad del ojo para acomodar la luz que le dirige sobre la propia retina, formando la imagen en sentido anterior y dificultando la visión lejana de los objetos.

1 Pontifícia Universidade Católica de Minas Gerais (PUC- MG), Betim - MG. *E-mail: laofpucmg@gmail.com ${ }^{2}$ Centro Oftalmológico de Minas Gerais (COMG), Belo Horizonte - MG. 
Esta patología tiene una prevalencia significativa en la infancia, constituyendo uno de los factores importantes para la baja agudeza visual. Al mismo tiempo, los primeros años de vida son los más importantes para el desarrollo del niño, por lo que la baja agudeza visual puede comprometer el desarrollo neuromotor, las habilidades manuales, el lenguaje y la cognición. Consideraciones finales: El desarrollo de las habilidades intelectuales de un niño es un proceso que depende de la información sensorial recibida a través de la visión. Así, identificar la baja agudeza visual en los escolares, en concreto la miopía, se vuelve fundamental para su correcto desarrollo cognitivo.

Palabras clave: Niño, Miopía, Cognición.

\section{INTRODUÇÃO}

Ver com qualidade é parte essencial da vida de todos os indivíduos, sendo a visão um importante fator do desenvolvimento habitual da criança (SANTOS MA, et al., 2021). Dentre as funções do olho, a capacidade de receber luz e a converter em impulsos elétricos destinados ao cérebro para formar as imagens que vemos é a principal responsável por permitir que habilidades intelectuais e sociais - psicomotora, cognitiva, locomotora e comunicativa - sejam desenvolvidas com maestria (VIEIRA JK, et al., 2018).

Estima-se que, no Brasil, a cada um milhão de pessoas, 280 mil são menores de 16 anos - faixa etária estabelecida pelo Fundo das Nações Unidas (UNICEF) como infância. É nesse período que ocorre o maior desenvolvimento das habilidades e associações visuais do indivíduo, principalmente até os 7 anos, que progridem segundo a idade cronológica. Entretanto, cerca de $20 \%$ das crianças que frequentam escolas apresentam baixa acuidade visual (PASCHE DF, et al., 2016).

As ametropias (astigmatismo, hipermetropia e miopia) são as principais causas da baixa acuidade visual nas crianças. A miopia, distúrbio frequente, é caracterizada pela incapacidade do olho de acomodar a luz direcionada a ele na própria retina, formando a imagem anteriormente, e dificultando a visão distante dos objetos (MAK CY, et al., 2018). Quando não corrigida de forma precoce, pode provocar consequências graves em todos os âmbitos da vida do indivíduo, incluindo deficiência visual (PEREIRA CF, et al., 2019).

Alguns fatores genéticos e ambientais estão em estudos e podem ser predisponentes à miopia, como hereditariedade, relação entre a pressão intraocular e a debilidade escleral, consumo de nicotina pelos pais, poder aquisitivo e o tempo gasto em uso de telas. Dessa forma, acredita-se que o tempo permanecido ao ar livre pode ser considerado importante fator protetor das ametropias (RAMAMURTHY D, et al., 2015).

Em tempos de pandemia, como o momento atual vivido em decorrência do COVID-19, o uso de telas tem sido o principal fator desencadeante das miopias. A necessidade de distanciamento social, de certa forma, exigiu que dispositivos como tablets, computadores e celulares fossem incorporados com maior frequência no dia a dia, principalmente pelo contexto escolar. Estudos mostram que entre 2019 e 2021, a miopia progrediu em $40 \%$ nos jovens de 5 a 18 anos. Esse crescimento pode ser encontrado também nas crianças pré-escolares. Isso se deve ao fato de que, além do uso escolar desses recursos, as crianças permanecem em uso também nos momentos de lazer, até mesmo para comodidade e segurança dos responsáveis (PICOTTI C, et al., 2021).

Considerando ainda que a maioria dessas crianças só descobrem a baixa acuidade visual e suas possíveis repercussões quando já estão nas escolas, a miopia é um problema de saúde pública. Para isso, no Brasil existem programas de triagem de acuidade visual realizada não só por oftalmologistas, mas também por outros profissionais capacitados, por meio da tabela de Snellen. E essa tabela permite avaliar apenas a acuidade visual. Quando observada alguma alteração na triagem, o paciente é encaminhado ao médico oftalmologista que é o responsável por realizar a avaliação completa, com possibilidade de detectar doenças, definir o grau do erro refracional e estabelecer a terapia adequada (SOUZA AG, et al., 2019).

Em vista de tudo isso, esse artigo objetiva evidenciar a importância de detectar e de tratar a miopia na criança de maneira precoce, evitando e/ou retardando a progressão da doença, contribuindo, assim, para a melhora do aproveitamento escolar e, principalmente, do desempenho neuropsicomotor do indivíduo. 


\section{REVISÃO BIBLIOGRÁFICA}

\section{Definição, etiologia e classificação}

A miopia é decorrente de um erro refrativo, em que os raios de luz que entram no olho paralelos ao eixo óptico convergem e focam antes da retina quando a acomodação visual está relaxada. Possui como causa principal o alongamento do eixo axial, mas também pode ser consequência de lentes com maior poder óptico ou uma córnea com uma curvatura excessiva. Aceita-se que miopia é um valor menor ou igual a $-0,5$ dioptrias de um erro esférico quando a acomodação está relaxada. Podemos classificar em graus variados a miopia de acordo com o valor de dioptrias do olho, sendo baixa entre $-0,5$ e $-6,0$ e alta para valores menores que $-6,0$ (WOLFFSOHN JS, et al., 2019).

Essa condição é resultado de um comprimento axial longo para o poder óptico de um olho. Caracterizada por visões borradas de objetos a distância e imagens mais nítidas dos mais próximos. Pode ter como etiologia uma forma sindrômica, que acomete outros tecidos do corpo de maneira congênita. Entretanto, a maioria das miopias não se enquadram nessa categoria e possuem um desenvolvimento de acordo com a idade de início, congênita (na infância, principalmente em crianças que nasceram prematuras), ou como produto de um crescimento desregulado do olho, nas faixas etárias juvenil e adulto, sendo a primeira a idade mais comum (HARB EM e WILDSON CF, 2019).

Estudos apontam que há uma interação entre a genética e o meio ambiente no desenvolvimento e na progressão da miopia. Em tais estudos e meta-análises, diversos genes foram identificados e relacionados, porém poucos foram replicados em grupos populacionais e quando isso foi possível, resultaram em uma pequena proporção de miopia. Evidências apontam uma relação maior entre fatores genéticos e a síndrome míope e miopia de alto grau. Com relação a miopia de baixo e de moderado grau acredita-se haver uma influência maior entre os fatores ambientais em comparação com os genéticos, além do aumento de sua prevalência em várias regiões do mundo (WOLFFSOHN JS, et al., 2019).

Considera-se que há fatores ambientais envolvidos com a miopia, contudo não existe uma elucidação completa de seus mecanismos. Os fatores que estão mais associados são nível socioeconômico mais elevado, maior escolaridade, vida em meio urbano e tempo reduzido ao ar livre (CHAKRABORTY R, et al, 2018).

O desenvolvimento da capacidade refrativa do olho passa por fases em direção à emetropia. Nos 6 primeiros meses de vida, os indivíduos apresentam, em sua maioria, um erro refrativo hipermetrope de grau baixo, que tende a emetropia até os 5 a 7 anos. De acordo com estudos, estima-se que a taxa de progressão da miopia é de $-0,5$ dioptrias ao ano a -1,0 D/ano entre as idades de 6 e 9 anos, na faixa etária acima de 10 a taxa varia entre $-0,35 \mathrm{D} / a n o$ e $-0,75 \mathrm{D} / \mathrm{ano}$. Tal progressão vai reduzindo com o avançar da idade na maioria dos pacientes e irá se estabilizar no final da adolescência, antes dos 20 anos. Contudo, há indivíduos que a miopia pode progredir durante os anos da vida adulta, essa forma está mais relacionada com fatores ambientais, destacam-se trabalhos intensos em uma distância próxima do nariz, como estudantes e aqueles que possuem níveis mais altos de miopia. A progressão na vida adulta e os altos graus associados tendem a levar o paciente a um risco maior de patologias visuais decorrentes da miopia (WOLFFSOHN JS, et al., 2019).

\section{Efeitos da miopia no desenvolvimento da criança}

A visão é um dos mais importantes sentidos no desenvolvimento normal da criança. Quando há o comprometimento dessa via sensorial, uma criança pode apresentar impactos no desenvolvimento neuropsicomotor, em suas relações familiares e ainda na realização de suas atividades básicas de vida (SANTOS MA, et al., 2021).

Os distúrbios de visão, são as causas mais frequentes de problemas de saúde nas escolas, sendo diretamente relacionados com o rendimento escolar. Em um estudo realizado na Colômbia, foi encontrado uma prevalência significativamente maior entre transtornos de visão entre alunos repetentes quando comparado com alunos não repetentes (VIEIRA JK, et al., 2018). Dessa forma, a detecção precoce desses distúrbios é fundamental, a fim de diminuir a dificuldade de aprendizado, os índices de reprovação e evasão escolar, o que vai gerar uma melhora no aprendizado escolar (SOUZA AG, et al., 2019). 
Por fim, as crianças que apresentam algum tipo de déficit de desempenho na área de autocuidado devido a visão, são mais dependentes de cuidadores pela dificuldade na mobilidade, além de dependerem também de uma pessoa para realizar suas atividades diárias básicas, diferente das crianças sem deficiência visual (SANTOS MA, et al., 2021).

\section{Fatores de risco para progressão da miopia na infância}

A miopia na criança é um distúrbio visual no qual tem seu risco aumentado por fatores biológicos e ambientais. Em geral, os estudos revisados abordaram as condições genéticas e hereditárias relacionandoas às demais questões que envolvem o meio da criança. Isso foi visto em casos, em que alguns fatores se mostraram predisponentes à doença apenas quando relacionados a outras condições ambientais. O sexo feminino, por exemplo, apresentou ter maior incidência e prevalência. Porém, a análise desse dado demanda uma associação aos hábitos tidos pelo gênero. Em um estudo realizado na Índia, foi relatado que meninas tendem a ler e a escrever mais e a passarem maior tempo em locais fechados, em relação aos meninos. Esse achado indica que o aumento dessa carga de estudo provavelmente influencia no desenvolvimento da miopia. A análise dos fatores de risco, portanto, deve ser feita englobando todo o contexto do paciente (SAXENA R, et al., 2017).

Entre os fatores biológicos, foi evidenciado associação significativa entre a história familiar e a miopia. Em um estudo transversal, realizado na Espanha, notou-se que, dependendo do número de pais portadores do distúrbio, a criança apresenta maior predisposição à doença. De forma que, o risco de ter miopia aumenta de $9,7 \%$, em um contexto no qual nenhum dos pais é míope, para $28,3 \%$, caso ambos forem. A idade também foi tida como fator de risco. Em geral, a prevalência da miopia aumentou com a progressão da idade. Esse dado pode estar associado ao fato de que o uso de dispositivos eletrônicos aumenta significativamente com a idade (ALVAREZ-PEREGRINA CC, et al., 2019).

Em relação às condições ambientais, a baixa exposição ao ar livre é também considerada fator de risco. De acordo com pesquisa realizada pelo Sydney Adolescent Vascular and Eye Study (SAVES), aquelas crianças que se tornaram míopes estiveram em menor tempo ao ar livre, em comparação com as que permaneceram não míopes. Isso porque a exposição à luz gera liberação de dopamina retiniana, responsável por controlar o crescimento e a remodelação escleral. Outros fatores incluem o tempo gasto com exercícios físicos e o alto peso corporal, nos quais estão diretamente associados ao tempo gasto em ambientes abertos (GRZYBOWSKI A, et al., 2020; ALVAREZ-PEREGRINA CC, et al., 2019; THEOPHANOUS C, et al., 2018).

Além desses fatores, a dedicação ao estudo se mostra com um risco recorrente à doença. Hábitos como o uso de lâmpadas LED influenciam nessa ocorrência. Em pesquisa realizada com 2.346 crianças chinesas, entre 13 e 14 anos, constatou-se que as crianças que faziam uso dessas lâmpadas, na realização de deveres escolares em casa, após o período na escola, apresentaram maior prevalência de miopia, bem como o comprimento axial maior, em relação àquelas que usavam lâmpadas incandescentes ou fluorescentes (PAN CW, et al., 2018).

Todo esse contexto também se associa à distância de leitura menor que 25 centímetros, que se mostrou influente à prevalência da Miopia. Essa prática é comum em atividades que envolvem ler, escrever, assistir à televisão, usar o telefone, o tablet ou o videogame. Em uma pesquisa com alunos do ensino fundamental e médio, na cidade de Guangzhou, as crianças que faziam leituras com distância menor que 25 centímetros, eram mais propensas a apresentar miopia, em relação às crianças que tinha o hábito de ler a distâncias de 25-29 ou mais centímetros. O tempo de estudo também foi analisado, sendo que, crianças que passaram lendo mais de duas horas diariamente foram positivamente associadas à doença. Essas relações podem ocorrer em razão do aumento da atividade visual próximas aos olhos ou da redução do tempo ao ar livre (GRZYBOWSKI A, et al., 2020; ALVAREZ-PEREGRINA CC, et al., 2019; GUO L, et al., 2016).

Foi visto que o fator socioeconômico da família e da comunidade em que as crianças estão inseridas estabelece um papel importante na determinação do risco da miopia. Estudos demonstram que aquelas crianças que fazem parte de um contexto familiar com renda mais alta apresentam um risco maior para desenvolvimento da doença. Essa condição pode ter relação com diferentes estilos de vida, como o tempo gasto com leituras e com aparelhos eletrônicos, bem como as atividades ao ar livre (THEOPHANOUS C, et al., 2018). 
Outro fator importante é o tempo de sono dessas crianças. Em um estudo, com 15.316 estudantes, de idade média de 12,1 $\pm 3,3$ anos de 18 distritos de Pequim, crianças com oito horas ou menos de sono tiveram um risco maior, comparadas àquelas que dormiam nove horas ou mais por dia. Sabe-se que a inatividade do músculo ciliar, durante o sono, tem a capacidade de prevenir ou aliviar a progressão miópica. Entretanto, esse mecanismo ainda não é bem descrito e outras variáveis podem estar envolvidas também, como a ação da luz fraca, por exemplo. Esse efeito sugere que as vias dos bastonetes, estimuladas pela exposição a essa luminosidade, podem se enquadrar como fatores importantes (GONG Y, et al., 2014; GRZYBOWSKI A, et al., 2020).

O uso de dispositivos eletrônicos foi positivamente relacionado à progressão da miopia. Ao longo dos anos, as mudanças de estilo de vida foram notadas entre as gerações. Atualmente, é notável que as crianças possuem maior contato com tecnologias. Isso vem preocupando médicos e educadores quanto ao risco potencial de ocorrência da miopia, o que pode impactar no desempenho acadêmico dessas crianças (ALVAREZ-PEREGRINA CC, et al., 2019; CHENG HG, et al., 2020).

Em um contexto atual, é importante destacar a potencial influência da pandemia do COVID-19. A partir das novas barreiras impostas pelo vírus, o fechamento generalizado das escolas fez com que as aulas fossem ministradas preferencialmente em ambiente virtual. Isso fez com que o tempo de tela digital aumentasse. Porém, apesar da tecnologia contribuir imensamente para diminuir os impactos na educação escolar, os efeitos a longo prazo na acuidade visual das crianças deve ser algo avaliado (WONG CW, et al., 2021).

As evidências atuais sobre esse impacto ainda são inconclusivas, no entanto, já se sabe que essa exposição pode contribuir no aumento da incidência de miopia mundialmente. A pandemia também impôs restrições de atividades ao ar livre, como forma de controlar a propagação do vírus, visando o distanciamento social. Essas políticas prejudicaram a exposição das crianças a ambientes abertos, fazendo com que elas passassem mais tempo em ambientes fechados (ENTHOVEN CA, et al., 2020; WONG CW, et al., 2021).

Por fim, é notório que muitos dos fatores de risco são potencialmente modificáveis. Esses devem ser alvos de esforços por parte da saúde pública, envolvendo medidas de proteção contra a miopia, de acordo com o contexto da criança e do meio que a envolve (GUO L, et al., 2016).

\section{Tratamento}

Tendo em vista os diversos fatores de riscos à ocorrência e à progressão da Miopia na infância, o tratamento precoce é extremamente importante para que intervenções invasivas não sejam necessárias. A prevenção de problemas visuais na infância requer um trabalho multidisciplinar, desde o nascimento até as fases pré-escolar e escolar, da atenção básica aos encaminhamentos especializados. As ações envolvem promoções de saúde, prevenção, diagnóstico e tratamento precoce (JIA QU, 2019).

Já ao nascimento, considerando a prevalência significativa de crianças que apresentam baixa acuidade visual por complicações na gestação no Brasil, o rastreamento visual ativo, pela avaliação ocular e pelo teste do reflexo vermelho, detecta alterações ópticas e possibilita a melhor conduta a ser adotada. Em períodos mais avançados existem inúmeros programas de rastreamento da visão infantil, como a desenvolvida pela Direção-Geral da Saúde que auxilia na qualidade de vida dessas crianças (MINISTÉRIO DA SAÚDE, 2010).

Para controlar o início e a progressão da miopia infantil alguns tratamentos são abordados para reduzir o aparecimento de miopia ou retardá-la. Uma forma de atrasar o aparecimento da miopia é aumentar o tempo com o olhar livre, sem usar a visão de perto. Em um estudo realizado na Austrália foi evidenciado que o tempo em que crianças míopes ficavam ao ar livre era menor quando comparado ao tempo das não míopes, sendo 16,3 horas por semana para as primeiras e, 21 horas semanais para as que não apresentaram miopia. $O$ estudo representa uma coorte de 863 crianças australianas (GINAC DB, 2020).

Uma maneira de postergar a progressão do distúrbio visual é o tratamento com atropina em doses reduzidas e a ortoqueratologia. A introdução de atropina diariamente impede $o$ afinamento ou alongamento da esclera e o crescimento do olho, causado por degenerações mióticas. Na pediatria, o tratamento com $0,025 \%$ de atropina para crianças reduz a progressão da miopia em $65 \%$, sem efeitos colaterais notórios que 
afetam a qualidade de vida delas, por 2 anos. Quanto à ortoceratologia, a finalidade é reduzir temporariamente a miopia com o uso de uma lente rígida de gás permeável que comprime a córnea diminuindo o raio de curvatura. A recomendação é que se utilize no período noturno e retire pela manhã. Como desvantagem há o risco de infecção, além da redução da oxigenação da córnea no período da noite (CUNHA JT, et al., 2018).

Ademais, existem também as lentes bifocais, progressivas e desfocadas que apesar de aparentemente não ter uma eficácia um estudo mostrou que houve uma redução do comprimento axial em $0,55 \mathrm{~mm}$ em comparação com crianças que usavam um óculos simples; e desfocagem de lentes de contato que através de um estudo duplo-cego randomizado também evidenciou uma diminuição de 0,32mm. É importante ressaltar que essas opções são melhores aceitas em conjunto com outras formas de tratamento (GINAC DB, 2020).

\section{CONSIDERAÇÕES FINAIS}

A baixa acuidade visual prejudica potencialmente o aprendizado e a capacidade de socializar da criança, provocando dificuldade e/ou atrasos no desenvolvimento de habilidades intelectuais e profissionais. Dessa forma, existe forte associação entre o desenvolvimento das competências intelectuais da criança e das informações sensoriais recebidas através da visão. E, por isso, identificar a baixa acuidade visual em escolares torna- se essencial para seu desenvolvimento psicomotor adequado. Crianças em ambiente escolar apresentam manifestações mais visíveis da perda visual, tornando possível e necessário o rastreio das ametropias, a fim de evitar progressão e maiores danos consequentes da visão subnormal da criança. Paralelamente, a intervenção precoce nestes quadros é determinante para o sucesso terapêutico e contribui para evitar a evolução destes.

\section{REFERÊNCIAS}

1. ALVAREZ-PEREGRINA CC, et al. Prevalence and Risk Factors of Myopia in Spain. Journal of Ophthalmology, 2019; 2019: 3419576.

2. CHAKRABORTY R, et al. Circadian rhythms, refractive development, and myopia. Ophthalmic \& Physiological Optics, 2018; 38: 217-245.

3. CHENG HC, et al. Risk Factors and Behaviours of Schoolchildren with Myopia in Taiwan. International Journal of Environmental Research and Public Health, 2020; 17(6): 1967.

4. CUNHA JT, et al. Diminuição da progressão da miopia com atropina 0,025\%. Rev. Bras. Oftalmol, 2018; 72-75.

5. ENTHOVEN CA, et al. The impact of computer use on myopia development in childhood: The Generation R study. Preventive Medicine, 2020; 132: 105988.

6. GARDON GAGLIARDO HG, NOBRE MI. Intervenção Precoce na Criança com Baixa Visão. Revista de Neurociências, 2001; 9(1): 16-19.

7. GIANINI RJ, et al. Prevalência de baixa acuidade visual em escolares da rede pública, Sorocaba. Rev Saúde Pública. 2004; 38(2):201-8.

8. GINAC DB. Myopie de l'enfant. M / S Journals, 2020; 763 - 768

9. GRZYBOWSKI A, et al. A review on the epidemiology of myopia in school children worldwide.BMC Ophthalmol, 2020; 20(1): 27.

10. GONG Y, et al. Parental myopia, near work, hours of sleep and myopia in Chinese children. Health, 2014; 6(1): 64-70.

11. GUO L, et al. Prevalence and associated factors of myopia among primary and middle school-aged students: a schoolbased study in Guangzhou. Eye (Lond), 2016; 30(6): 796-804.

12. HARB EN e WILDSON CF. Origins of Refractive Errors: Environmental and Genetic Factors. Annual Review Visual Science, 2019; 5:47-72.

13. JIA QU. Effective prevention and control of myopia in children and adolescents. Chin J Ophthalmol, 2019; 81-85.

14. MAK CY, et al. Epidemiology of myopia and prevention of myopia progression in children in East Asia: a review. Hong Kong Medical Journal, 2018; 24(6): 602-609.

15. MINISTÉRIO DA SAÚDE. Secretaria de Atenção à Saúde. Departamento de Atenção Básica. Rastreamento. Série A. Normas e Manuais Técnicos. Cadernos de Atenção Primária. 2010; (29):60.

16. PAN CW, et al. Types of Lamp for Homework and Myopia among Chinese School-Aged Children.Ophthalmic Epidemiology, 2018; 25(3): 250-256.

17. PASCHE DF, et al. Secretaria de Atenção à Saúde. Diretriz de Atenção à Saúde Ocular na Infância: detecção e intervenção precoce para a prevenção de deficiências visuais, 2016. 
18. PEREIRA CF, et al. Triagem de acuidade visual reduzida em uma unidade de Atenção Primária à Saúde. Revista Brasileira de Oftalmologia, 2019; 78(4): 250-254.

19. PICOTTI C, et al. Myopia progression in children during COVID-19 home confinement in Argentina. The Lancet, 2021; $14 \mathrm{p}$.

20. RAMAMURTHY D, et al. A review of environmental risk factors for myopia during early life, childhood and adolescence. Clinical and Experimental Optometry, 2015; 98(6): 496-506.

21. ROSA, J. "Qual é o impacto da 'epidemia de miopia' na produtividade global?". Revista Universo Visual, 2019.

22. SANTOS MA, et al. Desempenho funcional nas atividades básicas de vida diária em crianças com deficiência visual. Psicologia e Saúde em Debate, 2021; 7(2): 113-130.

23. SAXENA R, et al. Incidence and progression of myopia and associated factors in urban school children in Delhi: The North India Myopia Study (NIM Study). Plos One, 2017; 12(12): e0189774.

24. SECIN VK. Relações entre a Saúde e a Educação: as ciências da visão a favor da escola. Scielo books, 2020; 153168.

25. SOUZA AG, et al. Avaliação e triagem da acuidade visual em escolares da primeira infância. Revista Brasileira de Oftalmologia, 2019; 78(2): 112-116.

26. THEOPHANOUS C, et al. Myopia prevalence and risk factors in children. Clin Ophthalmol, 2018; $12: 1581-1587$.

27. VIEIRA JK, et al. Prevalência de baixa acuidade visual em escolares. Revista Brasileira de Oftalmologia, 2018; 77(4): 175-179.

28. WOLFFSOHN JS, et al. IMI - Myopia Control Reports Overview and Introduction. Investigative Ophthalmology \& Visual Science, 2019; 60: M1-M19.

29. WONG CW, et al. Digital Screen Time During the COVID-19 Pandemic: Risk for a Further Myopia Boom?. American Journal of Ophthalmology, 2021; 223: 333-337. 